\title{
Microcalcifications in breast cancer: an active phenomenon mediated by epithelial cells with mesenchymal characteristics
}

Manuel Scimeca', Elena Giannini', Chiara Antonacci ${ }^{1}$, Chiara Adriana Pistolese ${ }^{2}$, Luigi Giusto Spagnoli ${ }^{1}$ and Elena Bonanno ${ }^{1 *}$

\begin{abstract}
Background: Mammary microcalcifications have a crucial role in breast cancer detection, but the processes that induce their formation are unknown. Moreover, recent studies have described the occurrence of the epithelial-mesenchymal transition (EMT) in breast cancer, but its role is not defined. In this study, we hypothesized that epithelial cells acquire mesenchymal characteristics and become capable of producing breast microcalcifications.

Methods: Breast sample biopsies with microcalcifications underwent energy dispersive X-ray microanalysis to better define the elemental composition of the microcalcifications. Breast sample biopsies without microcalcifications were used as controls. The ultrastructural phenotype of breast cells near to calcium deposits was also investigated to verify EMT in relation to breast microcalcifications. The mesenchymal phenotype and tissue mineralization were studied by immunostaining for vimentin, BMP-2, $\beta 2$-microglobulin, $\beta$-catenin and osteopontin (OPN).

Results: The complex formation of calcium hydroxyapatite was strictly associated with malignant lesions whereas calcium-oxalate is mainly reported in benign lesions. Notably, for the first time, we observed the presence of magnesium-substituted hydroxyapatite, which was frequently noted in breast cancer but never found in benign lesions. Morphological studies demonstrated that epithelial cells with mesenchymal characteristics were significantly increased in infiltrating carcinomas with microcalcifications and in cells with ultrastructural features typical of osteoblasts close to microcalcifications. These data were strengthened by the rate of cells expressing molecules typically involved during physiological mineralization (i.e. BMP-2, OPN) that discriminated infiltrating carcinomas with microcalcifications from those without microcalcifications.

Conclusions: We found significant differences in the elemental composition of calcifications between benign and malignant lesions. Observations of cell phenotype led us to hypothesize that under specific stimuli, mammary cells, which despite retaining a minimal epithelial phenotype (confirmed by cytokeratin expression), may acquire some mesenchymal characteristics transforming themselves into cells with an osteoblast-like phenotype, and are able to contribute to the production of breast microcalcifications.
\end{abstract}

\section{Background}

Microcalcifications play a crucial role in early breast cancer diagnosis, the second leading cause of cancer death among women [1]. Approximately 50\% of non-palpable breast cancers are detected by mammography exclusively through microcalcification patterns [2], revealing up to $90 \%$ of ductal carcinoma in situ [3]. Mammary microcalcifications

\footnotetext{
* Correspondence: elena.bonanno@uniroma2.it

'Anatomic Pathology Section, Department of Biomedicine and Prevention, University of Rome "Tor Vergata", Via Montpellier 1, Rome 00133, Italy Full list of author information is available at the end of the article
}

are classified according to their mammographic morphology, i.e. density and distribution [4], and by their physical and chemical properties [5]. Type I calcifications are composed of calcium oxalate (CO), and are amber-colored, partially transparent, and form pyramidal structures with relatively planar surfaces. Type II calcifications are composed of calcium phosphate, mainly hydroxyapatite (HA); they are grey-white, opaque with ovoid or fusiform shapes and have irregular surfaces [5-7]. The mechanisms that induce the formation of microcalcifications in breast cancer are still unknown, and for a long period of time they have

\section{Biomed Central}


been considered a passive phenomenon [8]. Recently, it has been suggested that ectopic mineralization in pathological conditions might be regulated by mechanisms similar to those occurring in physiological conditions $[9,10]$. Calcification of bone during skeletal growth $[11,12]$ is sustained by mineralization-competent cells that are mesenchymal in origin, for example osteoblasts and hypertrophic chondrocytes [13], by three different processes: matrix vesiclemediated mineral initiation $[14,15]$, nucleation of mineral crystal [16,17] and ectopic mineralization [18].

Epithelial-mesenchymal transition (EMT), a complex phenomenon in which epithelial cells lose their characteristic traits and gain several properties of mesenchymal cells, is believed to play a role in breast cancer [19-21] and presents different changes at both the genetic and molecular level. EMT starts with the loss of cell polarity and the dissolution of tight junctions, allowing the intermingling of apical and basolateral membrane components [22]. Phenotypically, EMT involves the loss of epithelial cell markers such as E-cadherin and cytokeratin, and the acquisition of mesenchymal markers such as vimentin and nuclear $\beta$-catenin [23].

The first issue that we addressed in this study concerned the relationship between the elemental composition of calcification and the breast lesion type. The second approach was oriented to investigate if microcalcifications are related to an active process mediated by epithelial cells that enables acquisition of mesenchymal characteristics mimicking physiological mineralization.

To better define the phenomenon of microcalcifications, we took advantage of morphological characterization and microanalytical techniques correlating breast lesion types with the fine elemental composition of minerals. Furthermore, to assess a possible role of epithelial cells in tissue mineralization, we explored the cellular phenotype by correlating morphological data with molecular markers revealed by immunohistochemistry.

\section{Methods}

\section{Breast sample collection}

In this retrospective study, we collected 86 breast diagnostic biopsies in total: 60 vacuum-assisted needle biopsies, six surgical biopsies performed on radiologically suspicious breast microcalcifications and 20 samples of breast diagnostic biopsies without microcalcifications. Our study protocol was approved by the "Policlinico Tor Vergata" independent ethical committee (reference number \# 94.13).

\section{Histology}

After fixation in $10 \%$ buffered formalin for $24 \mathrm{~h}$, breast tissues were embedded in paraffin. Three-micrometerthick sections were stained with hematoxylin and eosin $(\mathrm{H} \& \mathrm{E})$ and the diagnostic classification was blindly performed by two pathologists [24].

\section{Tissue microarray (TMA)}

For TMA construction, we utilized fragments of tissues left over the sampling procedures for diagnostic purpose. Areas of interest from 20 infiltrating carcinomas without microcalcifications (ICwm) were identified in corresponding $\mathrm{H}$ \& E-stained sections and marked on the donor paraffin block. A 3-mm-thick core of the donor block was placed in the recipient master block of the Galileo TMA CK2500 (Brugherio, Milan, Italy). Three cores from different areas of the same tissue block were arrayed for each case (total amount of neoplastic cells not less than 1.500) [25].

\section{Immunohistochemistry}

Paraffin sections of 4- $\mu$ m-thick were cut both from diagnostic blocks and TMA, and were processed by the Bench Mark automatized system (Ventana, Tucson, AZ, USA). After pretreatment, sections were incubated with rabbit monoclonal anti-vimentin (clone V9; Ventana, Tucson, AZ, USA; pre-diluted) [26], rabbit monoclonal anti-bone morphogenic protein-2 (clone N/A; Novus Biologicals, Littleton, CO, USA; 1:500 diluted) [27], rabbit monoclonal anti- $\beta 2$ microglobulin (clone N/A; Dako Denmark A/S, Glostrup Denmark; 1:100 diluted) [28], rabbit monoclonal anti- $\beta$-catenin (clone 14; Ventana, Tucson, AZ, USA; prediluted) [29] and rabbit monoclonal anti-osteopontin (clone N/A; Novus Biologicals, Littleton, CO, USA; 1:100 diluted) [30] antibodies. Reactions were revealed with an ultraView Universal DAB Detection Kit (Ventana, Tucson, AZ, USA). For dual color immunohistochemistry, sections were stained using the same automatized system. Briefly, 4- $\mu \mathrm{m}-$ thick sections were pre-treated with $\mathrm{CC} 1$ reagent (Ventana, Tucson, AZ, USA) for $30 \mathrm{~min}$ at $95^{\circ} \mathrm{C}$ and then incubated with primary rabbit monoclonal anti-pan cytokeratin antibody for 20 min (clone AE1/AE3/PCK26; Ventana, Tucson, AZ, USA; pre-diluted). Reactions were revealed using an ultraView Universal DAB Detection Kit (Ventana, Tucson, AZ, USA). Sections were newly pre-treated with CC1 Ventana reagent for $8 \mathrm{~min}$ at $95^{\circ} \mathrm{C}$ and incubated with primary rabbit monoclonal anti-vimentin for $30 \mathrm{~min}$ (clone V9; Ventana, Tucson, AZ, USA). Vimentin reactions were revealed with an ultraView Universal Alkaline Phosphatase Red Detection Kit (Ventana, Tucson, AZ, USA).

\section{Transmission electron microscopy (TEM)}

Small pieces of breast tissue from surgical specimens were fixed in $4 \%$ paraformaldehyde, post-fixed in $2 \%$ osmium tetroxide [31] and embedded both in EPON resin and in London ResinWhite (LR-White) resin for morphological and immunoultrastructural studies. After washing with $0.1 \mathrm{M}$ phosphate buffer, the sample was dehydrated by a series of incubations in 30\%, 50\% and $70 \%$, ethanol. For EPON resin, dehydration was continued by incubation steps in $95 \%$ ethanol, absolute ethanol 
and propylene oxide, then samples were embedded in Epon (Agar Scientific, Stansted Essex CM24 8GF United Kingdom) [32].

For LR-White embedding (Agar Scientific, Stansted Essex CM24 8GF United Kingdom), dehydration was completed with incubations in 70\% ethanol-LR-White mixture (1:1) and LR-White absolute, then samples were embedded in LR-White resin [33]. After both types of incubation, tissues were cut $[34,35]$ and stained with heavy metals solutions as described by Reynolds [36].

\section{Energy dispersive $\mathrm{x}$-ray (EDX) microanalysis}

All breast samples underwent ultrastructural microanalysis. Six-micrometer-thick paraffin sections were embedded in Epon resin following identification of microcalcifications. Briefly, sections were deparaffinized, hydrated, osmium tetroxide-fixed, dehydrated in ethanol and propylene oxide and infiltrated in Epon. The embedding capsules were positioned over areas containing previously-identified microcalcifications. Unstained ultra-thin sections of approximately 100-nm-thick were mounted on copper grids for microanalysis. EDX spectra of microcalcifications were acquired with a Hitachi 7100FA transmission electron microscope (Hitachi, Schaumburg, IL, USA) and an EDX detector (Thermo Scientific, Waltham, MA, USA) at an acceleration voltage of $75 \mathrm{KeV}$ and magnification of 12.000. Spectra were semi-quantitatively analyzed by the Noram System Six software (Thermo Scientific, Waltham, MA, USA) using the standardless Cliff-Lorimer k-factor method [37]. EDX microanalysis apparatus was calibrated using an $\mathrm{x}$-ray microanalysis standard (Micro-Analysis Consultants Ltd., Cambridgeshire, UK).

\section{Immunogold labeling}

Ultrathin LR-White embedded sections, collected on Formvar carbon-coated nickel grids, were incubated in drops of $1 \%$ bovine serum albumin (BSA) in phosphate-buffered saline (PBS) containing $0.02 \mathrm{M}$ glycine and normal goat serum at room temperature for $30 \mathrm{~min}$ [38]. Sections were then incubated overnight with a rabbit monoclonal anti-vimentin antibody (clone V9; Ventana, Tucson, AZ, USA; pre-diluted) at $4^{\circ} \mathrm{C}$. After several washes with $\mathrm{PBS}+0.1 \% \mathrm{BSA}$, grids were incubated with a $20 \mathrm{~nm}$ secondary antibody-gold particle complex (Agar Scientific, Stansted Essex CM24 8GF United Kingdom) at 1:10 diluted in PBS 0.1\% BSA for $2 \mathrm{~h}$ at room temperature. After immunolabeling, sections were washed with $\mathrm{PBS}+0.1 \% \mathrm{BSA}$, washed in distilled water, dried, and counterstained with uranyl acetate. All sections were examined with a Hitachi 7100 FA electron microscope.

\section{Statistical analysis}

Statistical analysis was performed using GraphPad Prism 5 Software (La Jolla, CA, USA). Spatial distribution of microcalcifications within mammary lesions were analyzed by the Chi square test $(P<0.0001)$ to compare microcalcifications isotypes among $\mathrm{BLm}, \mathrm{ISCm}, \mathrm{ICm}$ and $\mathrm{ICwm}$ and by Fisher's exact tests $(P<0.0001)$ to analyze the associations between pairs of data sets.

Immunohistochemical data were analyzed by Kruskal-Wallis test $(P<0.0001)$ and by Mann-Whitney test $(P<0.0005)$.

\section{Results \\ Morphology}

Samples were classified as follows: 22 benign lesions (14 fibrocystic mastopathies and eight fibroadenomas) with microcalcifications (BLm), 21 ductal in situ carcinomas with microcalcifications (ISCm), 23 infiltrating ductal carcinomas with microcalcifications (ICm) and 20 infiltrating ductal carcinomas without microcalcifications (ICwm).

With regard to the morphology of microcalcifications, we found birefringent crystals in 14 BLm (eight fibrocystic mastopathies and six fibroadenomas), psammoma bodies in eight malignant lesions (seven ISCm and one $\mathrm{ICm}$ ), polymorphous bodies in both BLm (six fibrocystic mastopathies and two fibroadenomas) and malignant lesions (14 ISCm and $23 \mathrm{ICm}$ ) (see Additional file 1).

\section{Microcalcifications elemental analysis}

The ultrastructural elemental microanalysis performed on breast microcalcifications confirmed the presence of the already-known types of calcifications, $\mathrm{CO}$ and $\mathrm{HA}$ (Figure 1). In particular, CO microcalcifications appeared as unstained birefringent crystals in $79 \%$ of cases and as polymorphous bodies in $21 \%$ of cases; among the 24 HA microcalcifications, we observed seven psammoma bodies and 17 polymorphous bodies, whereas most of the magnesiumsubstituted hydroxyapatite (Mg-HAp) microcalcifications appeared as polymorphous bodies (22 polymorphous bodies and one psammoma body).

The presence of $\mathrm{CO}$ correlated with benign lesions in $81.8 \%$ of cases (18 out of 22 ), whereas $97.7 \%$ (43 out of 44) of malignant lesions were characterized by the presence of complex forms of microcalcifications (Figure 1). For the first time, EDX microanalysis allowed us to identify a new subtype of complex HA form, Mg-HAp (Figure 1E,F and $\mathrm{H}$ ). It is important to underline that Mg-HAp was detected only in malignant lesions (23 out of 44) whereas $\mathrm{CO}$ was never found in ICm (Figure 1).

\section{Epithelial cells undergoing mesenchymal transition}

Mesenchymal characteristics were assessed by vimentin and $\beta$-catenin detection. Immunohistochemical reactions were evaluated by counting the number of positive cells up to a total of 500 for each sample in a randomly-selected area containing microcalcifications (Figure $2 \mathrm{~B}-\mathrm{F}$ ). The rate of vimentin positive cells was significantly higher in malignant breast lesions with microcalcifications $(293.0 \pm 35.4$ in 



elemental composition of microcalcifications and breast lesions
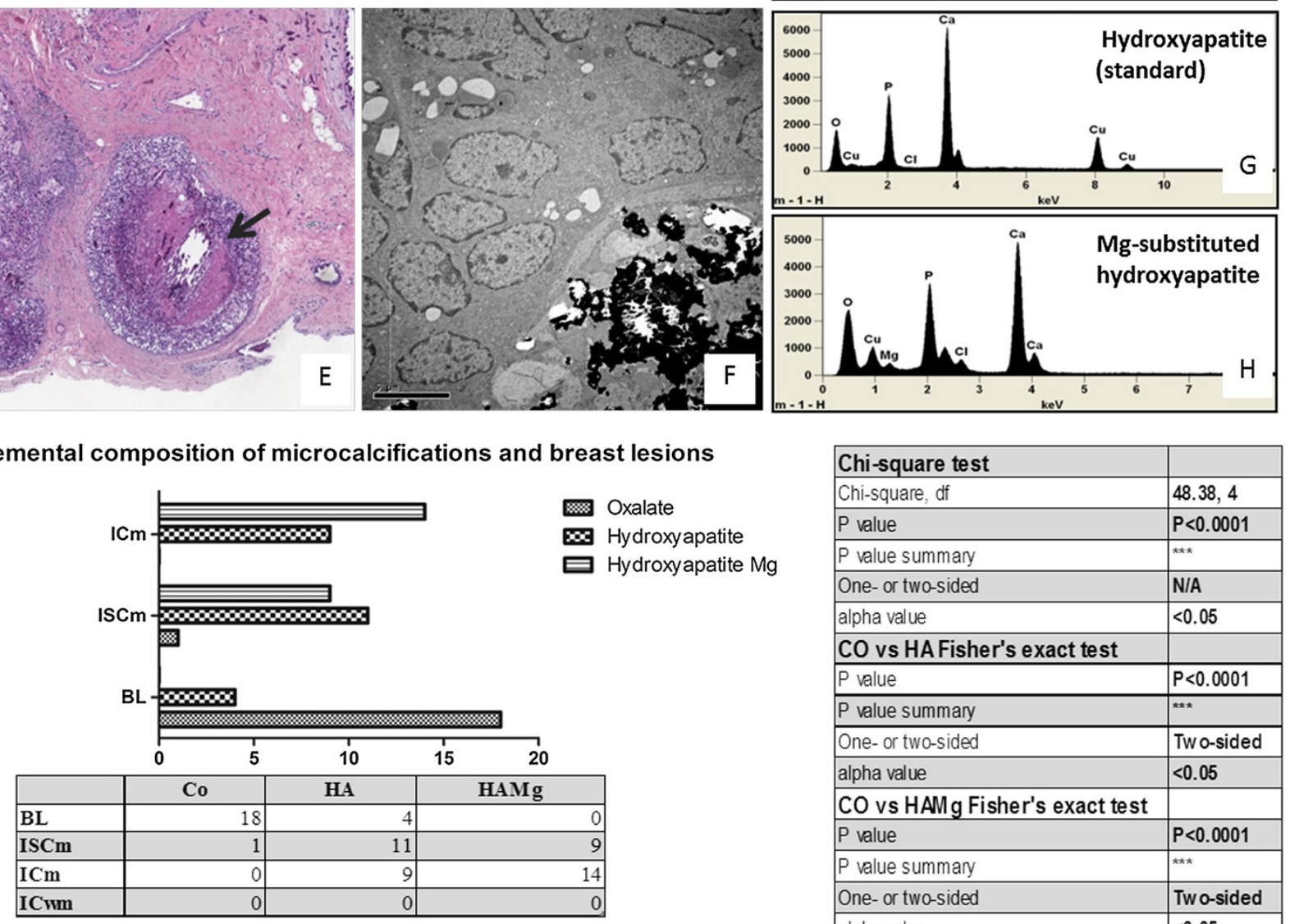

\begin{tabular}{|l|l|}
\hline Chi-square test & \\
\hline Chi-square, df & $48.38,4$ \\
\hline P value & $\mathrm{P}<0.0001$ \\
\hline P value summary & $* \star *$ \\
\hline One- or two-sided & N/A \\
\hline alpha value & $<0.05$ \\
\hline CO vs HA Fisher's exact test & \\
\hline P value & $\mathrm{P}<0.0001$ \\
\hline P value summary & $\star \star *$ \\
\hline One- or two-sided & Two-sided \\
\hline alpha value & $<0.05$ \\
\hline CO vs HAMg Fisher's exact test & \\
\hline P value & $\mathrm{P}<0.0001$ \\
\hline P value summary & $\star \star \star$ \\
\hline One- or two-sided & Two-sided \\
\hline alpha value & $<0.05$ \\
\hline
\end{tabular}

Figure 1 Elemental composition of calcification in breast pathology. (A) Microcalcifications (arrow) in BLm (fibroadenoma). (B) Electron micrograph by TEM of the microcalcification indicated in (A). (C) EDX spectra obtained by microanalysis of commercial standard sample utilized as a control. (D) EDX spectrum revealed that microcalcifications were composed of calcium oxalate (CO). (E) Microcalcifications (arrow) in an ISCm (comedocarcinoma). (F) Electron micrograph by TEM of the microcalcification indicated in (E). (G) EDX spectra obtained by microanalysis of commercial standard sample utilized as a control. (H) EDX spectrum revealed that this microcalcification was composed of magnesium-substituted hydroxyapatite (Mg-Hap). (I) Microcalcification type related to breast pathology by statistical analysis.

ICm; $116.9 \pm 38.9$ in ISCm) as compared with BLm (15.4 \pm 9.1) (Figure 3A).

Notably, we found that among infiltrating carcinomas, ICm showed a significantly higher number of vimentinpositive cells $(293.0 \pm 35.4)$ as compared with ICwm (162.1 \pm 33.7 ) (Figure 3A). We found the same trend when studying the translocation of $\beta$-catenin from the cytoplasmic membrane to the cytoplasm and to the nucleus (Figure 2D, $\mathrm{E}$ and F). Interestingly, we detected a strong increase in cells showing cytoplasmic/nuclear $\beta$-catenin staining in malignant lesions with microcalcifications (ICm 146.0 \pm 42.13 vs ICwm $59.83 \pm 20.11$ ) (Figure 3B).

The dramatically different rate of cells with vimentin and nuclear $\beta$-catenin expression in $\mathrm{ICm}$ as compared with 


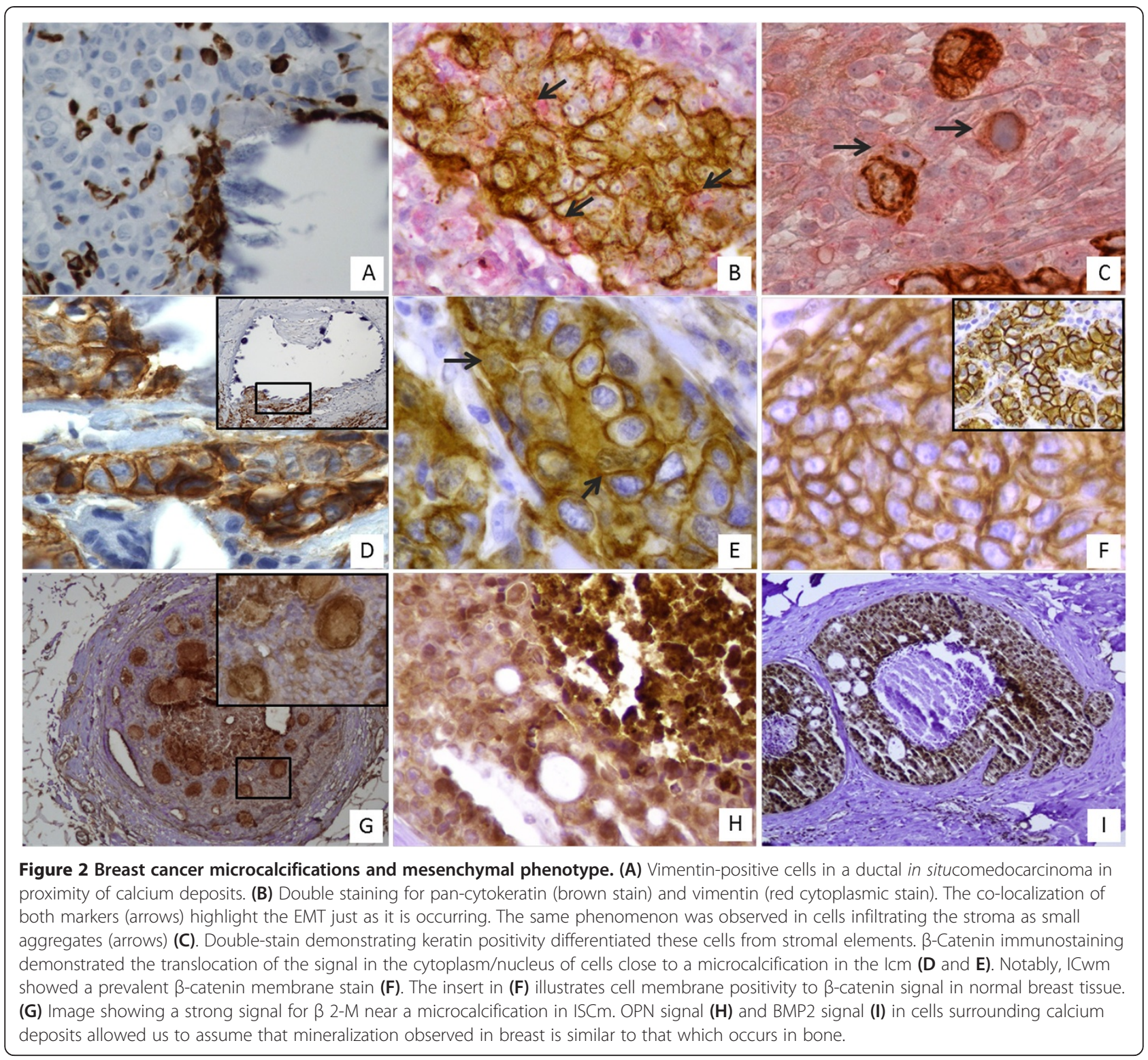

BLm and ICwm suggested that the formation of microcalcifications could be related to the EMT phenomenon (Figure 3A and B).

\section{Osteoblastic differentiation and mineralization}

Reactions for $\beta 2$-microglobulin ( $\beta 2-\mathrm{M})$, bone morphogenic protein-2 (BMP-2) and osteopontin (OPN) were evaluated by assigning a score from 1 to 3 according to the intensity of positive signals in randomly-selected regions (Figure 2G,H and I). As reported in Figure 3C, our results showed a striking increase in $\beta 2-\mathrm{M}$ signal in cancerous lesions with microcalcifications $(2.0 \pm 0.1)$ compared with both BLm $(0.5 \pm 0.1)$ and ICwm (1.5 \pm 1.1). Moreover, we demonstrated a significant difference in BMP-2 expression between infiltrating carcinomas with $(2.4 \pm 0.1)$ or without microcalcifications $(0.7 \pm 0.1)$ (Figure 3D).

The signal of OPN appeared very low in ICwm and homogenously widespread in BLm with CO microcalcifications (Figure 3E). In contrast, OPN showed a focal distribution with an increase in the signal in the proximity of $\mathrm{HA}$ and $\mathrm{Mg}$-HAp microcalcifications (Figure $2 \mathrm{H}$ ).

\section{Osteoblast-like cell characterization}

Our transmission electron microscopy study of cells located near HA and Mg-HAp microcalcifications revealed the presence of cells with morphological characteristics typical of osteoblasts (Figure 4). Osteoblast like-cells identified surrounding calcium deposits were positive for vimentin, as shown by immunogold labeling (Figure 4A, 
B and C). Their cytoplasms were rich in vesicles containing electron-dense granules similar to the intracellular vesicle of the osteoblasts (Figure $4 \mathrm{G}$ and $\mathrm{H}$; these intracellular vesicles were secreted outside cells (Figure 4G and $\mathrm{H}$ ). Elemental analysis of the electron-dense bodies inside these vesicles demonstrated the presence of HA (Figure 4I and J).

\section{Discussion}

Microcalcifications have a crucial role in breast cancer diagnosis. However, the mechanisms that induce their formation are still unknown [8]. In this paper, we investigated breast microcalcifications and hypothesized that they could result from a mineralization process similar to that of bone osteogenesis, sustained by the EMT phenomenon induced by microenvironmental stimulatory factors.

In our samples, we detect psammoma bodies in welldifferentiated carcinomas and polymorphous bodies both in ISCm and in ICm, as previously described [39]. Our data regarding the elemental composition of microcalcifications not only confirmed previous data about the presence of $\mathrm{CO}$ and HA [5-7] but most importantly allowed us to describe for the first time the presence of Mg-HAp in breast microcalcifications. It is important to underline that the complex forms of calcification (HA and Mg-HAp) are strictly related to malignant lesions whereas $\mathrm{CO}$ is mainly reported in benign lesions. Surprisingly, the EDX analysis revealed that some microcalcifications detected by light microscopy as polymorphous bodies were made of $\mathrm{CO}$. We postulated that this morphology could be due to a protein coat on the
CO crystal. Mg-HAp was not found in benign lesions whereas it was frequently detected in breast cancer. The capability of $\mathrm{HA}$ to bind to bicationic ions such as $\mathrm{Mg}$ [40], may confer carcinogenic properties on HA since a Mg-depleted microenvironment can influence the DNA repair processes and the control of proliferation and apoptosis $[41,42]$. The EDX data allowed us to hypothesize an active role of microcalcifications in breast carcinogenesis, since such complex microcalcifications cannot be due to a mere degenerative process but rather resemble the physiological process of mineralization that occurs in bone. At the same time, the presence of complex forms of calcification raises an interesting question: how can breast epithelial cells produce HA? Recently, Cox et al. [9,43] investigated the molecular mechanisms of the microcalcification process in breast cell cultures and demonstrated that the mineralization process, related to alkaline phosphatase activity, could be similar to that observed in bone matrix formation.

In response to these data, we investigated if the acquisition of mesenchymal characteristics could be a junction ring between breast epithelial cells and the complex microcalcifications that we described in breast cancer (Figure 5). Numerous studies have reported the EMT phenomenon in breast lesions [19,21] even though, to the best of our knowledge, no correlation of this phenomenon to calcium deposition has been made to date. Our data on mesenchymal markers (i.e. vimentin and $\beta$-catenin translocation) provided evidence that epithelial cells acquire mesenchymal characteristics in a process of mineralization in breast cancer. Indeed, the number of vimentin-positive cells was dramatically 

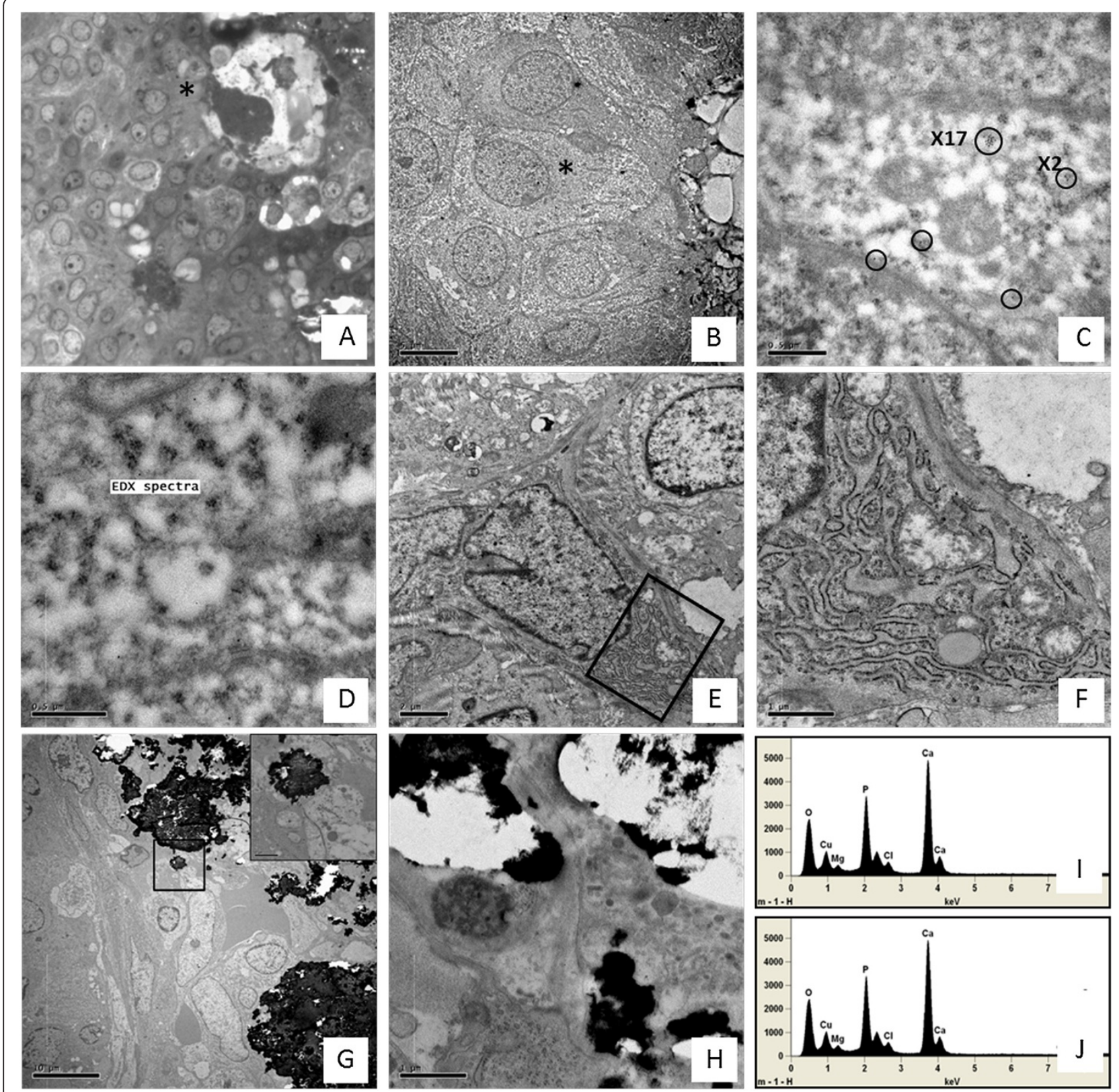

Figure 4 Osteoblast like-cell identification. Ultrastructural analysis of breast cancer cells surrounding microcalcifications showed some elongated cells (LR-White embedding). (A) Toluidine blue stain on a semi-thin section of cells surrounding microcalcification in malignant lesions. (B) Electron micrograph obtained by TEM of cells shown in (A). (C) Ultrastructural details of cells obtained by immunogold reaction for vimentin. Numerous gold particles indicated the presence of vimentin filaments inside the cytoplasm of this cell (circle). (D) Electron micrograph obtained by TEM on electron-dense intracytoplasmic bodies in the analyzed cell. EDX spectrum confirmed these consisted of HA crystals (I). (E), (F), (G) and $\mathbf{( H )}$ present finer ultrastructural analysis of breast cancer cells surrounding microcalcifications, as performed on Epon-embedded tissue. (E) and (F) show ultrastructural details of a cell exhibiting osteoblast-like morphology with a large cytoplasm and a huge rough reticulum. (G) Cell near a microcalcification containing a HA-matrix vesicle. The enlargement in $\mathbf{( G )}$ and $\mathbf{( H )}$ capture a matrix vesicle just as it initiated the exocytosis of HA (EDX spectrum in (I) and (J)).

different in infiltrating carcinomas with or without microcalcifications.

The results obtained by dual color immunohistochemistry for cytokeratin and vimentin markers further supported the evidence of mesenchymal transformation in
$\mathrm{ICm}$, since we observed cells in transition expressing both markers. In addition, these data were also sustained by the slight $\beta$-catenin translocation from the cytoplasmic membrane to cytoplasm and nuclei [44]. Furthermore, we examined some of the most important factors 


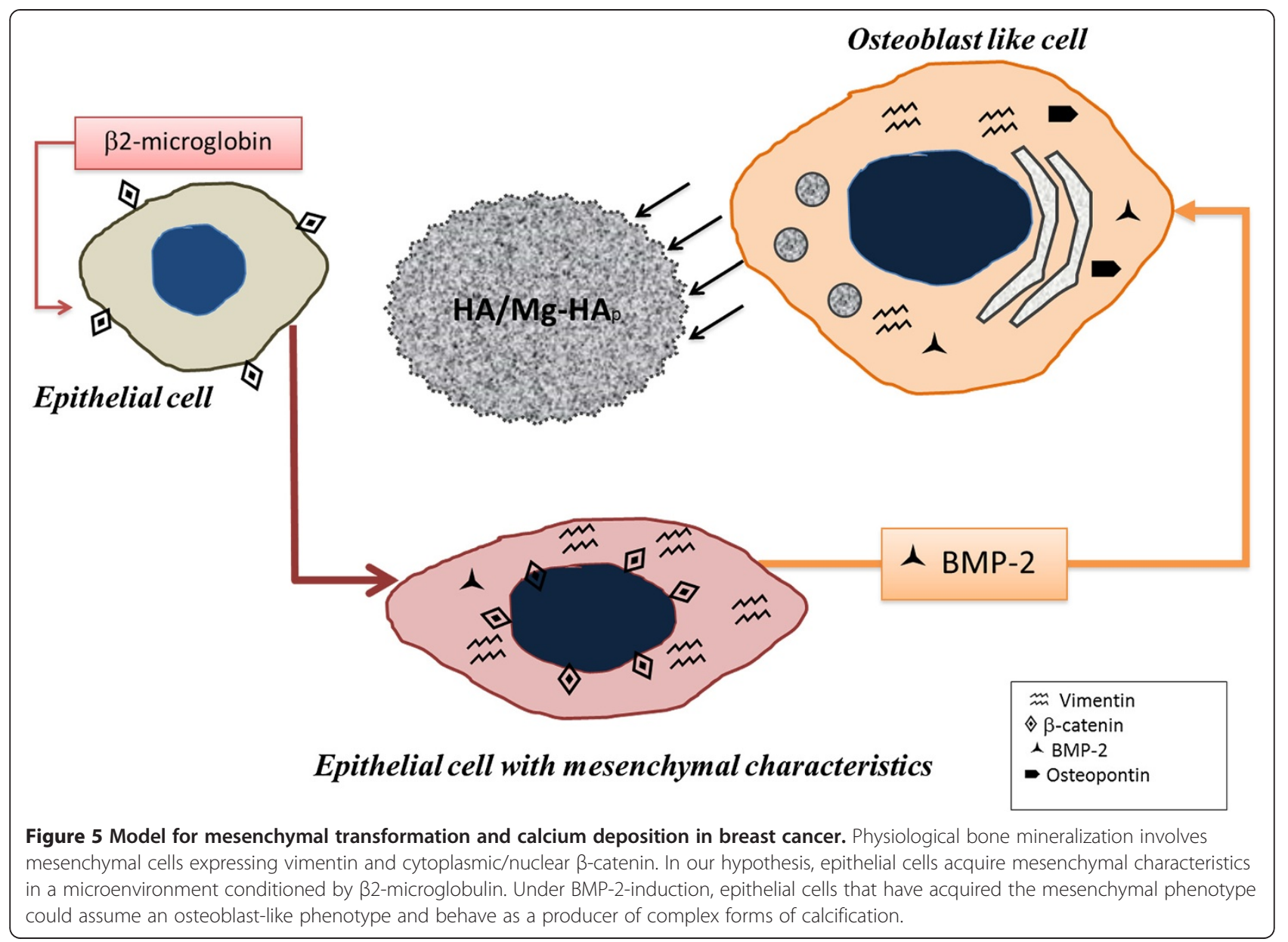

able to trigger the EMT. In particular, $\beta 2-\mathrm{M}$ was reported as a growth factor and signaling molecule in cancer cells [45-47]. It is also known to be able to trigger the EMT phenomenon as well as being capable of activating stromal cells, such as osteoblasts [48] and osteoclast [49]. The significant increase in this molecule in our breast cancer samples with microcalcifications suggests its possible role both in mesenchymal transformation and in the production of microcalcifications. To strengthen these data, we studied molecules involved in physiological bone mineralization. BMP2 is a member of the transforming growth factor superfamily and able to induce mineralization in osteoblasts cultures [50,51]. Furthermore, Liu et al. recently demonstrated a correlation between serum levels of BMP2 and breast microcalcifications [52]. Our results for the expression of BMP-2 in tissues with microcalcifications allowed us to assimilate the mineralization observed in the lesions of mammary glands with that occurring in osteoblast cultures, since both respond to the same signal.

Strikingly, when comparing in situ and infiltrating carcinomas with microcalcifications the signal appears to be intensively localized in the microenvironment surrounding the microcalcifications. OPN is another molecule that plays an important role in the mineralization process since it regulates both $\mathrm{HA}$ production [53] and its inhibition [54] depending on its phosphorylation state [55]. The data reported here on the expression of OPN suggest that it plays a role quite similar to that exerted during the physiological process of mineralization in bone [56,57].

Thus, such mineralization phenomenon in the context of the microcalcifications suggests the existence of cells able to produce HA. To provide proof of this conjecture, we performed an ultrastructural study in an attempt to identify cells with an osteoblast-like phenotype. At the ultrastructural level, the osteoblast is characterized by the presence of a well-developed rough endoplasmic reticulum with dilated cisternae and a dense granular content and by a large circular Golgi complex comprising multiple Golgi stacks [56]. The morphological characterization of cells surrounding the mineralized core displayed numerous cells exhibiting a mesenchymal phenotype surprisingly similar to osteoblasts. These osteoblast-like cells presented electrondense bodies in their cytoplasmic vesicles. The elemental characterization of these vesicles demonstrated that 
their content consisted of HA, a typical feature of osteoblast intracellular vesicles [58,59]. Taken together, these evidence led us to hypothesize that intracellular vesicles could be referred to as the center of nucleation of HA. Notably, we frequently observed osteoblast like cells secreting HA into the extracellular space. Finally, we confirmed by ultrastructural immunohistochemistry that these cells have a mesenchymal phenotype, as verified by their positivity to vimentin.

Although the phenomenon of breast microcalcifications could be sustained by several mechanisms, the finding of osteoblast-like cells led us to hypothesize that microcalcifications in breast lesions could represent an active process related to epithelial cells with mesenchymal characteristics.

\section{Conclusions}

New insights into the complex phenomenon of breast microcalcification could better define the pathophysiology of different microcalcifications. The introduction of mesenchymal markers such as vimentin and elemental analysis of breast lesions with microcalcifications may add further data to complete the clinical setting in the diagnosis and care of patients. The finding of a specific elemental composition associated with microcalcifications in cancer could enhance imaging technologies to discriminate microcalcifications in vivo, and thus act as a helpful tool in breast cancer screening.

\section{Additional file}

Additional file 1: Descriptive classification of microcalcifications in benign and malignant breast lesions. http://www.biomedcentral.com/ imedia/5099083231233250/supp1.pdf.

\section{Abbreviations}

BLm: Benign lesion with microcalcifications; BMP-2: Bone morphogenic protein; CO: Calcium oxalate; DAB: Diaminobenzidine; EDX: Energy dispersive x-ray; EMT: Epithelial to mesenchymal transition; H \& E: Hematoxylin and eosin; HA: Hydroxyapatite; HAMg: Hydroxyapatite magnesium substituted; ICm: Infiltrating carcinoma with microcalcifications; ICwm: Infiltrating carcinoma without microcalcifications; IHC: Immunohistochemistry; ISCm: In situ carcinoma with microcalcifications; Mg: Magnesium; OPN: Osteopontin; TMA: Tissue Micro-Array; $\beta 2-M$ : $\beta 2$-microglobulin.

\section{Competing interests}

The authors declare that they have no competing interests.

\section{Authors' contributions}

MS carried out the electron microscopy studies (EDX and colloidal gold studies), participated in the immunohistochemistry and drafted the manuscript. EG carried out the case selection and morphological classification. CA carried out immunohistochemistry. CAP participated in the design of the study and cases selection. LGS participated in the design of the study and cases selection. EB conceived of the study, and participated in its design, overall review of the result and coordination and helped to draft the manuscript. All authors were involved in writing the paper and had final approval of the submitted and published versions.

\section{Acknowledgements}

Authors wish to thanks Dr. Alessia Lucia Muzi, Dr. Simona Scano (University of Rome "Tor Vergata") and Francesca Della Gatta (University of Rome "Tor Vergata") for helpful discussion in planning stages of the work.

We acknowledge University of Tor Vergata for funding this study.

\section{Author details}

${ }^{1}$ Anatomic Pathology Section, Department of Biomedicine and Prevention, University of Rome "Tor Vergata", Via Montpellier 1, Rome 00133, Italy. ${ }^{2}$ Diagnostic Imaging Section, Department of Biomedicine and Prevention, University of Rome "Tor Vergata", Via Montpellier 1, Rome 00133, Italy.

Received: 5 September 2013 Accepted: 16 April 2014

Published: 23 April 2014

\section{References}

1. Jemal A, Sliegel R, Ward E, Murray T, Xu J, Thun MJ: Cancer statistics, 2007. CA Cancer J Clin 2007, 57:43-66.

2. Ferranti C, Coopmans de Yoldi G, Biganzoli E, Bergonzi S, Mariani L, Scaperrotta G, Marchesini M: Relationships between age, mammographic features and pathological tumour characteristics in non-palpable breast cancer. Br J Radiol 2000, 73(871):698-705.

3. Gülsün M, Demirkazik FB, Ariyürek M: Evaluation of breast microcalcifications according to Breast Imaging Reporting and Data System criteria and Le Gal's classification. Eur J Radiol 2003, 47(3):227-231.

4. Tabar L, Tony Chen HH, Amy Yen MF, Tot T, Tung TH, Chen LS, Chiu YH, Duffy SW, Smith RA: Mammographic tumor features can predict long-term outcomes reliably in women with 1-14-mm invasive breast carcinoma. Cancer 2004, 101(8):1745-1759.

5. Haka AS, Shafer-Peltier KE, Fitzmaurice M, Crowe J, Dasari RR, Feld MS: Identifying differences in microcalcifications in benign and malignant breast lesions by probing differences in their chemical composition using Raman spectroscopy. Cancer Res 2002, 62(18):5375-5380.

6. Frappart L, Boudeulle M, Boumendil J: Structure and composition of microcalcifications in benign and malignant lesions of the breast: study by light microscopy, transmission and scanning electron microscopy, microprobe analysis, and X-ray diffraction. Hum Pathol 1984, 15(9):880-889.

7. Radi MJ: Calcium oxalate crystals in breast biopsies. An overlooked form of microcalcification associated with benign breast disease. Arch Pathol Lab Med 1989, 113(12):1367-1369.

8. Busin GC, Keppler U, Menges V: Differences in microcalcification in breast tumours. Virchows Arch A Pathol Anat Histopathol 1981, 393:303-307.

9. Kirsch T: Determinants of pathological mineralization. Curr Opin Rheumatol 2006, 18(2):174-180.

10. Cox RF, Jenkinson A, Pohl K, O'Brien FJ, Morgan MP: Osteomimicry of mammary adenocarcinoma cells in vitro; increased expression of bone matrix proteins and proliferation within a $3 \mathrm{D}$ collagen environment. PLoS One 2012, 7(7):e41679.

11. Soltanoff CS, Yang S, Chen W, Li YP: Signaling networks that control the lineage commitment and differentiation of bone cells. Crit Rev Eukaryot Gene Expr 2009, 19(1):1-46.

12. Huang W, Yang S, Shao J, Li YP: Signaling and transcriptional regulation in osteoblast commitment and differentiation. Front Biosci 2007, 12:3068-3092

13. Anderson $\mathrm{HC}$ : Vesicles associated with calcification in the matrix of epiphyseal cartilage. J Cell Biol 1969, 41(1):59-72.

14. Anderson HC: Molecular biology of matrix vesicles. Clin Orthop Relat Res 1995, 314:266-280.

15. Anderson HC: Matrix vesicles and calcification. Curr Rheumatol Rep 2003, 5(3):222-226.

16. Landis WJ, Glimcher MJ: Electron optical and analytical observations of rat growth plate cartilage prepared by ultracryomicrotomy: the failure to detect a mineral phase in matrix vesicles and the identification of heterodispersed particles as the initial solid phase of calcium phosphate deposited in the extracellular matrix. J Ultrastruct Res 1982, 78(3):227-268.

17. Ghadially FN: As you like it, Part 3: A critique and historical review of calcification as seen with the electron microscope. Ultrastruct Pathol 2001, 25(3):243-267.

18. Marsh ME, Munne AM, Vogel JJ, Cui Y, Franceschi RT: Mineralization of bone-like extracellular matrix in the absence of functional osteoblasts. J Bone Miner Res 1995, 10(11):1635-1643. 
19. Mani SA, Guo W, Liao MJ, Eaton EN, Ayyanan A, Zhou AY, Brooks M, Reinhard F, Zhang CC, Shipitsin M, Campbell LL, Polyak K, Brisken C, Yang J, Weinberg RA: The epithelial-mesenchymal transition generates cells with properties of stem cells. Cell 2008, 133(4):704-715.

20. Morel AP, Lièvre M, Thomas C, Hinkal G, Ansieau S, Puisieux A: Generation of breast cancer stem cells through epithelial-mesenchymal transition. PLoS One 2008, 3(8):e2888.

21. Creighton CJ, Chang JC, Rosen JM: Epithelial-mesenchymal transition (EMT) in tumor-initiating cells and its clinical implications in breast cancer. J Mammary Gland Biol Neoplasia 2010, 15(2):253-260.

22. Godde NJ, Galea RC, Elsum IA, Humbert PO: Cell polarity in motion: redefining mammary tissue organization through EMT and cell polarity transitions. J Mammary Gland Biol Neoplasia 2010, 15(2):149-168.

23. Thiery JP, Acloque H, Huang RY, Nieto MA: Epithelial-mesenchymal transitions in development and disease. Cell 2009, 139(5):871-890.

24. Fox $\mathrm{CH}$, Johnson FB, Whiting J, Roller PP: Formaldehyde fixation. $J$ Histochem Cytochem 1985, 33:845-853.

25. Pilla D, Bosisio FM, Marotta R, Faggi S, Forlani P, Falavigna M, Biunno I, Martella E, De Blasio P, Borghesi S, Cattoretti G: Tissue microarray design and construction for scientific, industrial and diagnostic use. J Pathol Inform 2012, 3:42.

26. Benjamin E, Law S, Bobrow LG: Intermediate filaments cytokeratin and vimentin in ovarian sex cord-stromal tumours with correlative studies in adult and fetal ovaries. J Pathol 1987, 152(4):253-263.

27. Torihashi S, Hattori T, Hasegawa H, Kurahashi M, Ogaeri T, Fujimoto T: The expression and crucial roles of BMP signaling in development of smooth muscle progenitor cells in the mouse embryonic gut. Differentiation 2009, 77(3):277-289

28. Solé M, Muñoz-Gómez J, Campistol JM: Role of amyloid in dialysis-related arthropathies. A morphological analysis of 23 cases. Virchows Arch A Pathol Anat Histopathol 1990, 417(6):523-528.

29. Montgomery E, Torbenson MS, Kaushal M, Fisher C, Abraham SC: Beta-catenin immunohistochemistry separates mesenteric fibromatosis from gastrointestinal stromal tumor and sclerosing mesenteritis. Am J Surg Pathol 2002, 26(10):1296-1301.

30. Hapangama DK, Raju RS, Valentijn AJ, Barraclough D, Hart A, Turner MA, Platt-Higgins A, Barraclough R, Rudland PS: Aberrant expression of metastasis-inducing proteins in ectopic and matched eutopic endometrium of women with endometriosis: implications for the pathogenesis of endometriosis. Hum Reprod 2012, 27(2):394-407.

31. Hayat MA: Fixation for electron microscopy. New York: Academic; 1981.

32. Luft JH: Improvements in epoxy resin embedding methods. J Biophys Biochem Cytol 1961, 9:409-414.

33. Larsson LI: Simultaneous ultrastructural demonstration of multiple peptides in endocrine cells by a novel immunocytochemical method. Nature 1979, 282(5740):743-746.

34. Reid N: Ultramicrotomy. In Practical methods in electron microscopy, Volume 3. Amsterdam: North Holland; 1975. Part 2.

35. Dykstra MJ: Biological electron microscopy: theory, techniques and troubleshooting. New York: Plenum Press; 1992.

36. Reynolds ES: The use of lead citrate at high $\mathrm{pH}$ as an electron opaque stain based on metal chelation. J Cell Biol 1963, 17:208-212.

37. Scimeca M, Anemona L, Colantoni A, Bonanno E: Ultrastructural and microanalytical study of a subcutaneous granuloma HPV tetravalent vaccine induced: a case report and review of literature. J Med Case 2013, 4(7):461-465.

38. Perrotta I, Carito V, Russo E, Tripepi S, Aquila S, Donato G: Macrophage autophagy and oxidative stress: an ultrastructural and immunoelectron microscopical study. Oxid Med Cell Longev 2011, 2011:282739.

39. Foschini MP, Fornelli A, Peterse JL, Mignani S, Eusebi V: Microcalcifications in ductal carcinoma in situ of the breast: histochemical and immunohistochemical study. Hum Pathol 1996, 27(2):178-183.

40. Laurencin D, Almora-Barrios N, de Leeuw NH, Gervais C, Bonhomme C, Mauri F, Chrzanowski W, Knowles JC, Newport RJ, Wong A, Gan Z, Smith ME: Magnesium incorporation into hydroxyapatite. Biomaterials 2011, 32(7):1826-1837.

41. Castiglioni S, Maier JA: Magnesium and cancer: a dangerous liason. Magnes Res 2011, 24(3):S92-S100.

42. Wolf Fl, Trapani $\mathrm{V}$ : Magnesium and its transporters in cancer: a novel paradigm in tumour development. Clin Sci (Lond) 2012, 123(7):417-427
43. Cox RF, Hernandez-Santana A, Ramdass S, McMahon G, Harmey JH, Morgan MP: Microcalcifications in breast cancer: novel insights into the molecular mechanism and functional consequence of mammary mineralisation. Br J Cancer 2012, 106(3):525-537.

44. Kim K, Lu Z, Hay ED: Direct evidence for a role of beta-catenin/LEF-1 signaling pathway in induction of EMT. Cell Biol Int 2002, 26(5):463-476.

45. Huang WC, Havel JJ, Zhau HE, Qian WP, Lue HW, Chu CY, Nomura T, Chung LW: Beta2-microglobulin signaling blockade inhibited androgen receptor axis and caused apoptosis in human prostate cancer cells. Clin Cancer Res 2008, 14(17):5341-5347.

46. Rowley DR, Dang TD, McBride L, Gerdes MJ, Lu B, Larsen M: Beta-2 microglobulin is mitogenic to PC-3 prostatic carcinoma cells and antagonistic to transforming growth factor beta 1 action. Cancer Res 1995, 55(4):781-786.

47. Shuster J, Gold P, Poulik MD: Beta 2-microglogulin levels in cancerous and other disease states. Clin Chim Acta 1976, 67(3):307-313.

48. Evans DB, Thavarajah $M$, Kanis JA: Immunoreactivity and proliferative actions of beta 2 microglobulin on human bone-derived cells in vitro. Biochem Biophys Res Commun 1991, 175(3):795-803.

49. Menaa C, Esser E, Sprague SM: Beta2-microglobulin stimulates osteoclast formation. Kidney Int 2008, 73(11):1275-1281.

50. zur Nieden NI, Kempka G, Rancourt DE, Ahr HJ: Induction of chondro-, osteo- and adipogenesis in embryonic stem cells by bone morphogenetic protein-2: effect of cofactors on differentiating lineages. BMC Dev Biol 2005, 5:1

51. Suzuki A, Ghayor C, Guicheux J, Magne D, Quillard S, Kakita A, Ono Y, Miura $Y$, Oiso $Y$, Itoh M, Caverzasio J: Enhanced expression of the inorganic phosphate transporter Pit-1 is involved in BMP-2-induced matrix mineralization in osteoblast-like cells. J Bone Miner Res 2006, 21(5):674-683.

52. Liu F, Bloch N, Bhushan KR, De Grand AM, Tanaka E, Solazzo S, Mertyna PM, Goldberg N, Frangioni JV, Lenkinski RE: Humoral bone morphogenetic protein 2 is sufficient for inducing breast cancer microcalcification. Mol Imaging 2008, 7(4):175-186

53. McKee MD, Nanci A: Osteopontin: an interfacial extracellular matrix protein in mineralized tissues. Connect Tissue Res 1996, 35(1-4):197-205.

54. Boskey AL, Maresca M, Ullrich W, Doty SB, Butler WT, Prince CW: Osteopontin hydroxyapatite interactions in vitro: inhibition of hydroxyapatite formation and growth in a gelatin-gel. Bone Miner 1993, 22:147-159.

55. Gericke A, Qin C, Spevak L, Fujimoto Y, Butler WT, Sørensen ES, Boskey AL: Importance of phosphorylation for osteopontin regulation of biomineralization. Calcif Tissue Int 2005, 77:45-54.

56. Ecarot-Charrier B, Shepard N, Charette G, Grynpas M, Glorieux FH: Mineralization in osteoblast cultures: a light and electron microscopic study. Bone 1988, 9(3):147-154.

57. Silbermann M, Lewinson D: An electron microscopic study of the premineralizing zone of the condylar cartilage of the mouse mandible. J Anat 1978, 125:55-70.

58. Howlett CR: The fine structure of the proximal growth plate of the avian tibia. J Anat 1979, 128:377-399.

59. Rohde M, Mayer $\mathrm{H}$ : Exocytotic process as a novel model for mineralization by osteoblasts in vitro and in vivo determined by electron microscopic analysis. Calcif Tissue Int 2007, 80:323-336.

doi:10.1186/1471-2407-14-286

Cite this article as: Scimeca et al:: Microcalcifications in breast cancer: an active phenomenon mediated by epithelial cells with mesenchymal characteristics. BMC Cancer 2014 14:286 